\title{
Inheritable effect of unpredictable maternal separation on behavioral responses in mice
}

\author{
Isabelle C. Weiss ${ }^{\dagger}$, Tamara B. Franklin' ${ }^{\dagger}$ Sándor Vizi and Isabelle M. Mansuy*
}

Brain Research Institute, University of Zurich/Swiss Federal Institute of Technology, Zurich, Switzerland

\section{Edited by:}

Nora Abrous, Institut des

Neurosciences de Bordeaux, France

\section{Reviewed by:}

Catherine Belzung, Université François

Rabelais, France

Muriel Darnaudery, University of Lille

1, France

\section{*Correspondence:}

Isabelle M. Mansuy, Medical Faculty of the University of Zurich and

Department of Biology, Swiss Federal Institute of Technology,

Winterthurerstrasse 190, CH-8057

Zurich, Switzerland

e-mail:mansuy@hifo.uzh.ch

${ }^{+}$Isabelle C. Weiss and Tamara B.

Franklin have contributed equally to this work.
The long-term impact of early stress on behavior and emotions is well documented in humans, and can be modeled in experimental animals. In mice, maternal separation during early postnatal development induces poor and disorganized maternal care, and results in behavioral deficits that persist through adulthood. Here, we examined the long-term effect of unpredictable maternal separation combined with maternal stress on behavior and its transmissibility. We report that unpredictable maternal separation from birth to postnatal day 14 in C57BI/6J mice has mild behavioral effects in the animals when adult, but that its combination with maternal stress exacerbates this effect. Further, the behavioral deficits are transmitted to the following generation through females, an effect that is independent of maternal care and is not affected by cross-fostering. The combined manipulation does not alter basic components of the hypothalamic-pituitary-adrenal axis but decreases the expression of the corticotropin releasing factor receptor 2 (CRFR2) in several nuclei of the amygdala and the hypothalamus in the brain of maternal-separated females. These results suggest a non-genomic mode of transmission of the impact of early stress in mice.

Keywords: unpredictable maternal separation, inheritance, epigenetic, corticotropin releasing factor receptor

\section{INTRODUCTION}

The environment that an individual is exposed to in early life strongly influences the development of behavioral responses in adulthood. While this influence has been largely recognized, its extent and nature remain not well defined, and have been the subject of much debate. Many clinical studies have provided evidence that detrimental factors such as early abuse or trauma can have a severe impact on behaviors, and continue to affect individuals into and throughout their adult life. Thus, maltreatment, neglect, and trauma during childhood are known to increase the risk of psychiatric diseases such as depression and anxiety disorders in adulthood (Jaffee et al., 2002; Iversen et al., 2007; Moffitt et al., 2007; Heim et al., 2008; Rikhye et al., 2008; Neigh et al., 2009). Moreover, a strong link concerning the development and expression of such disorders between parent and offspring, and a high degree of transmission have been reported. Such transmission cannot purely result from parental factors, but instead has been postulated to derive from a predisposition of the offspring to the disease mediated by a combination of genetic and non-genetic factors (Hirshfeld-Becker et al., 2004; Shamir-Essakow et al., 2005).

The long-term impact of early trauma has been examined in rodent models of early stress using maternal separation (MS) paradigms that model perturbed mother-infant interaction, early life deprivation, and/or neglect (for review, see Holmes et al., 2005).

\footnotetext{
Abbreviations: BLA, basolateral amygdala; BMA, basomedial amygdala; $\mathrm{CPu}$, caudate putamen; CRFR, corticotropin releasing factor receptor; DG, dentate gyrus; Ect, ectorhinal cortex; LH, lateral hypothalamus; MePV/D, medial posteroventral and medial posterodorsal amygdala; MSU, unpredictable maternal separation; MSUS, unpredictable maternal separation combined with unpredictable stress; PRh, perirhinal cortex; PVN, paraventricular nucleus; $\mathrm{S} 1 / \mathrm{S} 2$, somatosensory cortex.
}

MS procedures all involve maternal deprivation but can vary in duration (1-24h) or number of separations during the first 2 weeks following delivery. Further, rearing conditions of the control group may also differ, creating differences and sometimes inconsistencies between paradigms. Thus, while some MS paradigms were reported to induce persistent anxiety and depressive-like behaviors, and changes in the hypothalamic-pituitary-adrenal axis (HPA axis) response to stressful environments (Huot et al., 2001; Lehmann et al., 2002; Parfitt et al., 2004; Murgatroyd et al., 2009), others were reported to lead to risk-taking and novelty-seeking behaviors in rats and mice (McIntosh et al., 1999; Colorado et al., 2006; Roman et al., 2006; Slotten et al., 2006; Fabricius et al., 2008; Mathieu et al., 2008; Franklin et al., 2010). These differences between paradigms may result from differences in the overall level of stress produced in the pups, but also in the dams exposed to MS. The effects of MS are thought to directly result from the physical separation of the dam from her pups, and also be mediated by the perturbation of maternal behaviors. In rodents, some of the direct effects of MS, for instance alterations of the immune system, increased neuronal cell death or hypersensitivity of the HPA axis, were shown to be reversed by artificial stroking during separation or by providing dams with a foster litter (Huot et al., 2004). These observations indicated that the effects of MS are mediated by the stress on both the dam and her pups. The impact of the separation on the dam not only includes alterations in maternal behavior, but also physiological parameters such as the level of stress hormones in the milk.

To examine how perturbations of the emotional state of a mother influence behavioral responses in the offspring, we developed a paradigm for MS in mice that is applied alone or in combination with unpredictable maternal stress. Here, we demonstrate that the addition of unpredictable maternal stress to unpredictable maternal 
separation (MSU) aggravates the effect of the manipulation on behavioral responses in the offspring. With this additional stress, the effects of the separation are transmitted to the following offspring. Transmission occurs through females, and is independent of maternal behavior, suggesting a non-genomic mode of transmission. We further show that key mediators of the stress response are differentially altered by the manipulation. While HPA axis markers are not altered, the density of corticotropin releasing factor receptor 2 (CRFR2) but not CRFR1 is decreased in hypothalamic and amygdala regions in the brain. The behavioral effects of the manipulation are similar for the dams and the pups, suggesting that comparable mechanisms may be engaged.

\section{MATERIALS AND METHODS \\ ANIMALS}

C57Bl/6J females and males (21/2 months) were obtained from Elevage Janvier (Le Genest Saint Isle, France) and maintained in a temperature- and humidity-controlled facility on a 12-h reverse light/dark cycle with food and water ad libitum. All procedures were carried out in accordance with Swiss cantonal regulations for animal experimentation.

\section{MATERNAL SEPARATION}

Unpredictable maternal separation and unpredictable maternal separation combined with unpredictable maternal stress (MSUS) dams $(F 0)$ and litters $(F 1)$ were subjected to separation for $3 \mathrm{~h}$ per day from postnatal day 1 (PND1) to PND14. Control mice were left undisturbed except for a cage change once a week until weaning (PND21). MSU, MSUS, and control dams and litters had their cages changed on PND1, PND7, PND14, and PND21, during which time the pups were also weighed. During separation, mothers and pups were placed in separate clean cages containing food and water (dams only), and bedding. Pups remained together during the separation period, and temperature during this time was not controlled. Litters and dams were placed such that they had visual and olfactory contact. The timing of separation was unpredictable, but was always during the dark cycle. In MSUS, maternal stress consisted of either 20-min restraint in a Plexiglas tube or 5-min forced swim in cold water $\left(18^{\circ} \mathrm{C}\right)$ applied unpredictably and randomly during the 3-h separation from the pups. Only dams giving birth within 1 week were used. An example of an MSU/MSUS schedule is provided in Table $\mathbf{1}$ (dark/light cycle: 8:00 h/20:00 h).

Table $\mathbf{1}$ | Example of a MSU/MSUS schedule during the first
postnatal week.
\begin{tabular}{llll}
\hline Day & MS & $\begin{array}{l}\text { Type of maternal } \\
\text { stress (MSUS only) }\end{array}$ & $\begin{array}{l}\text { Time of maternal } \\
\text { stress (MSUS only) }\end{array}$ \\
\hline & & & $13: 35-13: 55$ \\
2 & $11: 15-14: 15$ & Restraint & $11: 05-11: 10$ \\
3 & $11: 00-14: 00$ & Forced swim & $12: 00-12: 20$ \\
4 & $9: 30-12: 30$ & Restraint & $13: 10-13: 30$ \\
5 & $11: 00-14: 00$ & Restraint & $13: 30-13: 35$ \\
6 & $12: 15-15: 15$ & Forced swim & $14: 00-14: 20$ \\
7 & $13: 30-16: 30$ & Restraint & $14: 00-14: 05$
\end{tabular}

For maternal care scoring, dams and litters were observed once every minute for a total of $30 \mathrm{~min}$, and their behavior was monitored. This was done three times daily between PND1 and PND7, immediately before the separation, immediately after the separation, and $2 \mathrm{~h}$ following separation for $F 0$ dams, and at three time points randomly chosen throughout the day for $F 1$ dams. Both active and passive maternal behaviors were recorded. Active behaviors included arched-back nursing (ABN), licking and grooming (LG), ABN + LG (ABN-LG), nest building alone, ABN + nest building, blanket nursing, carrying pups, and self-grooming (Caldji et al., 2000; Liu et al., 2000). Percentage of incidences of off-nest behavior was recorded as a measure of reduced maternal care. All scoring took place during the dams' active cycle (dark phase of the light cycle).

Once weaned, pups were reared in same-sex social groups (3-4 mice/cage) composed of animals subjected to the same treatment but from different dams to avoid litter effects. To produce a second generation, female $F 1$ control and MSUS mice were mated to naïve C57Bl6/J males. For cross-fostering, F2 control or MSUS offspring was raised by $F 1$ MSUS or control dams, respectively. F2 offspring were weaned at PND21 and reared in mixed social groups similarly to F1. Litters were not culled.

\section{BEHAVIORAL TESTING}

In all tests, the experimenter was blind to treatment, and behaviors were monitored by direct observation, and videotracking (Ethovision, Noldus Information Technology). Behaviors were assessed in adult $F 0, F 1$, and $F 2$ animals (3-8 months old). Mice were tested on no more than three behavioral tests, 1-2 weeks apart, starting with the least aversive task, under indirect dim red light. To avoid possible litter effects, $F 1$ mice were randomly selected from a total of nine MSU, nine MSUS, and nine control litters. F2 mice were randomly selected from 11 MSUS and 15 control litters raised with their natural dams, five MSUS litters raised with control dams, and five control litters raised with MSUS dams. The number of pups per litter and the sex ratio of each litter were similar in all groups (Tables 2-5).

Table 2 | Number of pups/litter in F1 mice.

\begin{tabular}{lll}
\hline & Mean \pm SEM & Number of litters \\
\hline Control & $5.0 \pm 0.65$ & 9 \\
MSU & $4.5 \pm 0.44$ & 9 \\
MSUS & $5.2 \pm 0.52$ & 9 \\
\hline
\end{tabular}

Litter treatment: $F(2,24)=0.39, p=0.68, n s$.

Table 3 | Sex ratio (male/female) within litters of $\boldsymbol{F} 1$ mice.

\begin{tabular}{lll}
\hline & Mean \pm SEM & Number of litters \\
\hline Control & $0.88 \pm 0.12$ & 8 \\
MSU & $1.21 \pm 0.43$ & 8 \\
MSUS & $1.32 \pm 0.39$ & 9
\end{tabular}

Litter treatment: $F(2,22)=0.45, p=0.64, n s$. 
Table 4 | Number of pups/litter in F2 mice.

\begin{tabular}{lll}
\hline & Mean \pm SEM & Number of litters \\
\hline Control litter-control dam & $6.0 \pm 0.56$ & 15 \\
Control litter-MSUS dam & $7.0 \pm 0.55$ & 5 \\
MSUS litter-control dam & $7.2 \pm 0.86$ & 5 \\
MSUS litter-MSUS dam & $5.6 \pm 0.54$ & 11 \\
\hline
\end{tabular}

Litter treatment: $F(1,32)=0.013, p=0.91, n s$.

Dam treatment: $F(1,32)=0.15, p=0.70$, ns.

Table 5 | Sex ratio (male/female) within litters of $\boldsymbol{F} \mathbf{2}$ mice.

\begin{tabular}{lll}
\hline & Mean \pm SEM & Number of litters \\
\hline Control litter-control dam & $1.54 \pm 0.21$ & 15 \\
Control litter-MSUS dam & $1.24 \pm 0.37$ & 5 \\
MSUS litter-control dam & $2.58 \pm 1.47$ & 4 \\
MSUS litter-MSUS dam & $1.55 \pm 0.46$ & 9 \\
\hline
\end{tabular}

Litter treatment: $F(1,29)=1.584, p=0.22$, ns.

Dam treatment: $F(1,29)=1.54, p=0.23$, ns.

\section{Free exploratory paradigm}

$F 1$ and F2 MSUS and control mice were tested on the free exploratory paradigm (Griebel et al., 1993; Teixeira-Silva et al., 2009). The test consists of a small box $(31.5 \mathrm{~cm} \times 21 \mathrm{~cm} \times 20.5 \mathrm{~cm})$ containing fresh bedding with two rows of three-square partitions that are all connected. Mice were habituated to three connected partitions for $24 \mathrm{~h}$ with food and water ad libitum. Testing began when mice were allowed access to the row of unfamiliar partitions for $10 \mathrm{~min}$ exploration. The number of partition crossings was quantified using an automatic scoring system (Ethovision, Noldus Information Technology), and latency to enter into the unfamiliar area, attempts, and rearing were scored manually.

\section{Open field and open field emergence test}

F1 MSUS and control mice were tested in the open field and open field emergence test (Birke and Sadler, 1986; Quartermain et al., 1996). The tests consist of an open field $(50 \mathrm{~cm} \times 50 \mathrm{~cm} \times 30 \mathrm{~cm})$ with (open field emergence test) or without (open field) a home box $(20 \mathrm{~cm} \times 20 \mathrm{~cm} \times 14 \mathrm{~cm})$. In the case of the open field emergence test, mice were habituated to the home box for $24 \mathrm{~h}$ with bedding, food, and water ad libitum. The home box was then connected to one corner of the open field and access was provided for $10 \mathrm{~min}$. Time spent in the center of the open field in the open field emergence test, and total distance covered in the open field test, were quantified using an automatic scoring system (Ethovision, Noldus Information Technology). Latency to enter the open field and attempts to enter the open field in the open field emergence test were scored manually.

\section{Elevated plus maze}

$F 0, F 1$, and F2 MSUS and control mice were tested in the elevated plus maze. Mice were placed for $5 \mathrm{~min}$ on a four-arm plus maze made of two open and two closed arms (dark gray PVC, $30 \mathrm{~cm} \times 5 \mathrm{~cm}$ ) raised $60 \mathrm{~cm}$ above the ground. Manual scoring was done to quantify stretch attends in protected (body in closed arm) versus unprotected (body in open arm) areas as a measure of risk assessment. Time spent in the open arms and percentage of distance covered in the open arms were quantified using an automatic scoring system (Ethovision, Noldus Information Technology).

\section{PLASMA CORTICOSTERONE ASSAY}

Blood was collected from tail artery after scalpel incision then rapidly centrifuged in lithium/heparin-coated tubes (15 I.U.heparin/ $\mathrm{ml}$ blood, Microvette ${ }^{\varpi}$, Sevelen) at $6000 \mathrm{rpm}$ at $4^{\circ} \mathrm{C}$ for $5 \mathrm{~min}$. Plasma was collected and stored at $-80^{\circ} \mathrm{C}$ until used for radioimmunoassay. Blood was sampled 1 week before stress, immediately after stress (20-min restraint in a Plexiglas tube under white light), and $1 \mathrm{~h}$ after stress. Plasma immunoreactive corticosterone titers were quantified using a modified kit (Coat-A-Count Rat Corticosterone, Diagnostic Product Corp., Buehlmann Laboratories AG). Fifty microliters of plasma were used for assays. Standards (0-2 ng/ $\mathrm{ml}$ ) and samples were measured in duplicate. Tubes were vortexed and incubated at room temperature for $2 \mathrm{~h}$. The liquid tracer was then aspired off followed by 30 -s centrifugation at $2000 \mathrm{rpm}$ and removal of the remaining liquid. Radioactivity was measured for 1 min using a Wallac Wizard 1470 Gamma Counter (PerkinElmer). Assay validation yielded an inter-assay coefficient of variation of $14.9,5.8$, and $4.8 \%$ (calculated from 20 incubations of pairs of tubes for each of three samples of $27.5,161$, and $421 \mathrm{ng} / \mathrm{ml}$, respectively) and intra-assay coefficient of variation was $12.2,4.3$, and $4.0 \%$ (calculated from 20 pairs of tubes for each of three samples of $24.5,164$, and $427 \mathrm{ng} / \mathrm{ml}$, respectively). The sensitivity was approximately $5.7 \mathrm{ng} / \mathrm{ml}$.

\section{CRFR BINDING}

Corticotropin releasing factor receptor binding was carried out according to Tezval et al. (2004). The brain of F1 female MSUS and control adult mice was extracted, embedded in Shandon Cryomatrix (Thermo) and frozen at $-80^{\circ} \mathrm{C}$. Serial coronal sections $(20 \mu \mathrm{m})$ were cut at Bregma -0.58 to -0.94 and -1.22 to -1.46 . To confirm Bregma positions during cutting, occasional sections were Nissl stained, magnified, and controlled for the right position. Coronal sections were mounted onto APES-coated slides and stored at $-80^{\circ} \mathrm{C}$. Sections were preincubated in incubation buffer (PBS pH 7.2 with $10 \mathrm{mM} \mathrm{MgCl}_{2}$, 2 mM EGTA, 0.1\% BSA) for $1 \mathrm{~min}$ at room temperature. For CRFR1 binding, sections were treated in incubation buffer containing $200 \mathrm{pM}$ $\left[{ }^{125} \mathrm{I}_{-} \mathrm{Tyr}^{\circ}{ }^{\circ}\right.$ Sauvagine (a non-specific CRFR ligand; PerkinElmer Life Sciences) and, for selective displacement, $1 \mu \mathrm{M}$ mouse UrocortinII (a CRFR2 specific agonist, Phoenix Pharmaceuticals). For CRFR2 binding, the incubation buffer contained $100 \mathrm{pM}\left[{ }^{125} \mathrm{I}-\mathrm{His}^{2}\right]$ Antisauvagine-30 (a CRFR2 specific ligand; GE Healthcare/Amersham). Non-specific binding (NSB) was determined in the presence of $1 \mu \mathrm{M}$ Sauvagine (Phoenix). Sections were washed in ice-cold PBS pH 7.2 $0.01 \%$ Triton X-100 and in ice-cold water then air-dried. As control, CRFR binding was also performed with $200 \mathrm{pM}\left[{ }^{125} \mathrm{I}_{-} \mathrm{Tyr}^{\circ}\right]$ Sauvagine (and with $1 \mu \mathrm{M}$ Sauvagine for NSB) on sections from CRFR1 and CRFR2 knock-out mice (kindly provided by Dr. Jan Deussing; data not shown). Radioactively labeled sections were exposed to SR phosphor screens (Packard) for $24 \mathrm{~h}$ at room temperature. After exposure, sections were Nissl stained with $2 \%$ cresyl violet and brain areas of interest were identified and outlined using a mouse brain atlas 
(Paxinos and Franklin, 2001). Photographs of Nissl stained sections with outlined regions were then overlapped with corresponding phosphorimages in Photoshop CS2 (Adobe) and the phosphorimages were quantified using OptiQuant 4.0 (Packard). Labeling values of brain regions were corrected for screen background and NSB and expressed in net digital light unit $(\mathrm{dlu}) / \mathrm{mm}^{2}$ units. Typically, for each animal and each brain region, four measurements were performed on two sections. Means of net labeling values were calculated for each brain region in each animal.

\section{STATISTICAL ANALYSES}

All behavioral data in F1 control, MSU, and MSUS mice were analyzed using one-way ANOVAs followed by Fisher's PLSD post hoc. All behavioral data, weight, and maternal care scoring in F2 mice were analyzed using a $2 \times 2$ ANOVA (dam treatment $\times$ pup treatment) followed by a Bonferroni post hoc, when appropriate. Pearson correlation coefficients between maternal care and dam behavior were calculated, and statistical significance was determined using a correlation $z$-test. CRFR binding was analyzed using unpaired $t$-tests within each brain area. $F 1$ pup weight across development and plasma corticosterone were analyzed using a repeated-measures ANOVA. All data analyzed matched the requirements for parametric statistical tests. Significance was set at $p<0.05$ for all tests. Error bars represent SEM. $* p<0.05, * * p<0.01, * * * p<0.001$.

\section{RESULTS}

\section{UNPREDICTABLE MATERNAL SEPARATION ALTERS DAM BEHAVIOR}

Maternal separation was applied in two conditions with increasing severity, MSU and MSUS, to investigate the effect of additional unpredictable maternal stress during separation. Behavioral responses in adult $F 1$ mice were examined on several paradigms with gradually increasing degree of stress and aversion.

The analyses of $F 1$ MSU and MSUS litters showed that they grew normally, and had normal body weight when adult, despite being separated from their mothers and receiving perturbed maternal care (as seen by impaired maternal behaviors, for MSUS conditions see Franklin et al., 2010; Figure 1A). We further examined the effect of the most severe treatment, MSUS, on separated dams. F0 MSUS dams had reduced body weight compared to control dams during the period of separation, both after 7 and 14 days (PND7 and PND14). However, they recovered 1 week after the end of separation [PND21; Figure 1B; $F(3,84)=6.92, p<0.01]$. The stress associated with the separation also mildly perturbed the dams' behavior up to 1 month after the end of separation. When tested on the elevated plus maze, F0 MSUS dams tended to spend more time in the open arms $[t(14)=1.94, p=0.073]$ and covered a greater proportion of their total distance in these arms $[t(14)=2.01, p=0.064$; Figures 1C,E]. Further, the amount of time spent off-nest positively correlated with the time spent $\left(r^{2}=0.72, p<0.05\right)$, and percent distance covered in the open arms $\left(r^{2}=0.72, p<0.05\right)$ in the control group (Figures 1D,F). However, this correlation was not observed in the MSUS group (time: $r^{2}=0.50$, ns; distance $r^{2}=0.20$, ns).

\section{BEHAVIORAL ALTERATIONS INDUCED BY MS IN ADULT F1 MICE}

To evaluate the impact of the manipulation on adult behavior, F1 control, MSU, and MSUS adult mice were first tested under mildly stressful conditions using the free exploratory paradigm

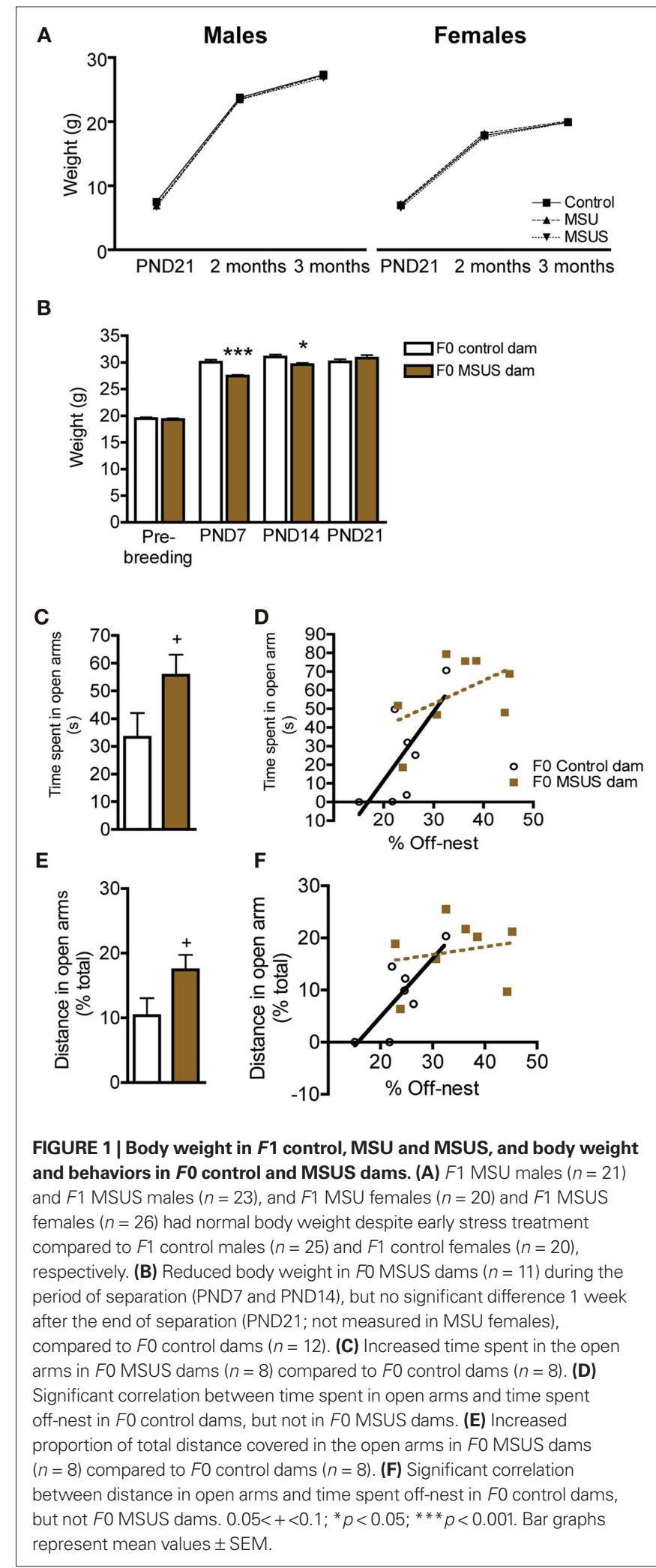

(Griebel et al., 1993). This task assesses exploratory and risk assessment behaviors by measuring the animals' response to an unfamiliar versus familiar environment. Before entering an unfamiliar environment, rodents typically evaluate the potential danger 
associated with unfamiliarity by making tentative entries and by assessing risk. MSUS resulted in a mild decrease in these behaviors associated with reduced careful exploratory assessment compared to control mice. No overall significant effect in latency to enter the unfamiliar areas was observed in $F 1$ males or $F 1$ females (Figure 2A). However, a strong trend toward a decrease in latency to enter the unfamiliar areas was observed in F1 MSUS males and F1 MSU females. There was also a significant effect of MS treatment in attempts to enter the unfamiliar partitions of the task, as MSUS males and MSU females made significantly less attempts to enter the unfamiliar partitions of the task [male: $F(2,41)=4.13, p<0.05$; female MSU: $F(2,38)=3.48, p<0.05$; Figure 2B]. This was not due to an altered locomotor activity or overall arousal since all $F 1$ animals had comparable numbers of partition crossings and rears during testing (Figures 2C,D).

On a slightly more aversive task, the open field emergence test (Birke and Sadler, 1986; Quartermain et al., 1996), a similar reduction in careful behaviors was observed in F1 MSU males and MSUS animals. No overall significant effect in latency to enter the novel open field from a familiar enclosure was observed in $F 1$ males or $F 1$ females. However, similar to the free exploratory paradigm, a strong trend toward a reduced latency to enter the novel open field was observed in MSUS, but not MSU, males (Figure 3A). Furthermore, there was a significant effect of MS on time spent in the most aversive area [the center of the open field; $F(2,19)=4.00, p<0.05$ ], and in number of exploratory attempts before entering the open field $[F(2,19)=3.83, p<0.05$; Figures 3B,C $]$. Post hoc analyses showed that this main effect was due to a significant increase of the time that MSUS males spent in the center of the open field, and a significant decrease in exploratory attempts before entering the open field. MSU males showed similar, but non-significant difference in these two parameters. The effect was more pronounced in MSUS than MSU males, clearly demonstrating the graded impact of treatment, with the addition of maternal stress enhancing the behavioral alterations. The effect was however not seen in females (Figure 3). To again confirm that activity was not altered in these animals, control, MSU, and MSUS mice were tested in an open field. Similar to the free exploratory paradigm, MSU and MSUS mice had normal locomotor activity, and covered a similar distance to control mice in the open field (Figure 3D), indicating no alteration in basal activity.

We next tested $F 1$ control, MSU, and MSUS animals on a highly aversive task, the four-arm elevated plus maze (Pellow et al., 1985; Lister, 1987). A main effect of MS treatment on time spent in open arms was observed [males: $F(2,39)=2.84, p=0.071$; females: $F(2$, $20)=6.78, p<0.01]$. Post hoc analyses showed that this effect was significant in F1 MSUS, but not MSU, males and females (Figure 4A). Additionally, a strong trend was observed in the proportion of the total distance covered in the open arms in males, since F1 MSUS males, but not $F 1$ MSU males, covered a greater proportion of the total distance in the open arms $[F(2,39)=3.20, p=0.052$; Figure 4B]. Both MSU and MSUS males made more unprotected stretch attends (with their body in open areas) than control animals $[F(2,39)=7.19, p<0.01$; Figure $4 \mathrm{C}]$, indicating reduced caution (Rodgers and Dalvi, 1997). Similar to the emergence test, the overall effect of MSU and MSUS was minor in females, although some behavioral parameters, such as time spent in the open arms, were

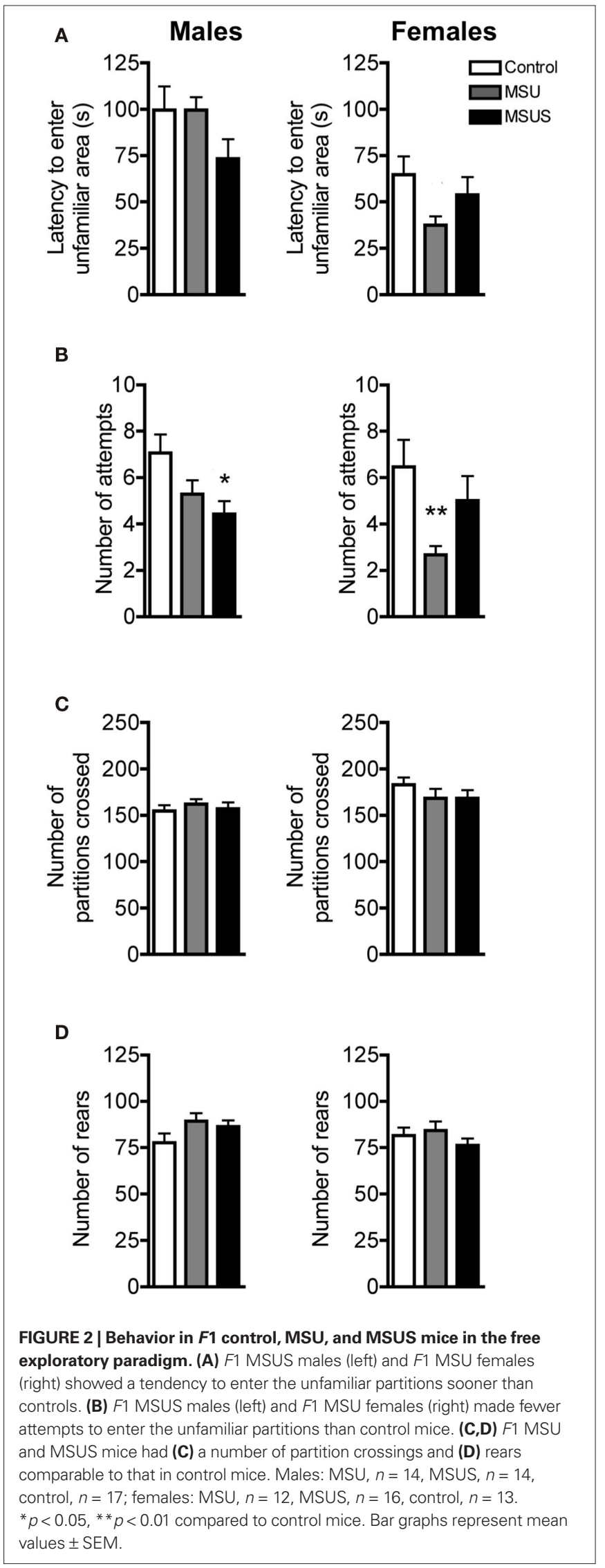



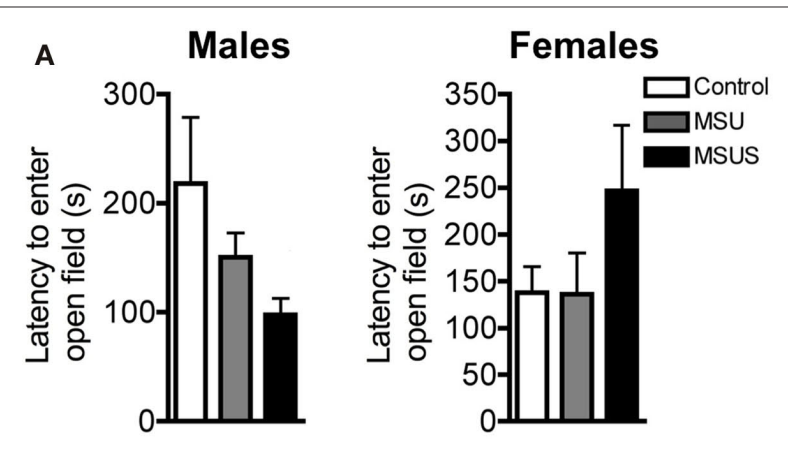

B
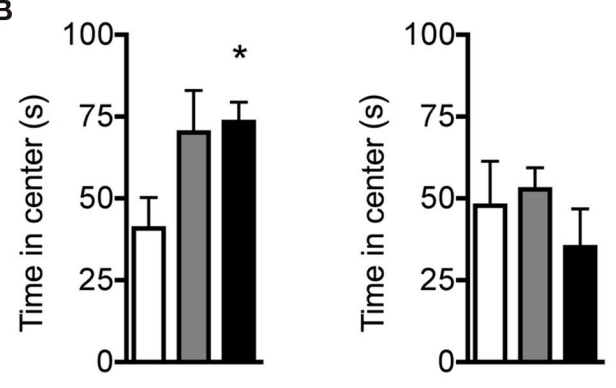

C
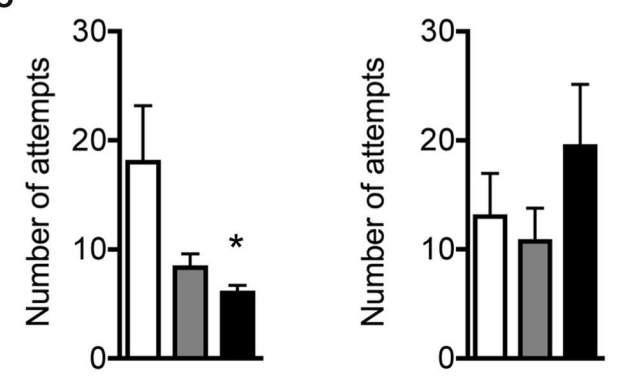

D
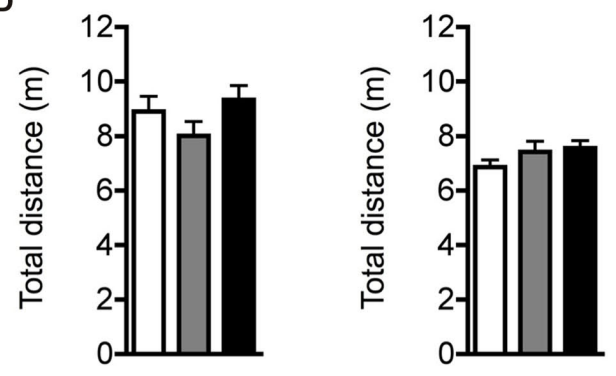

FIGURE 3 | Behavior in $\mathbf{F 1}$ control, MSU, and MSUS mice in the open field emergence test. (A) F1 MSU and MSUS males had a graded decrease in latency to first enter the open field (left), but F1 MSU and MSUS females had a latency comparable to control mice (right). (B) F1 MSUS males spent more time in the center of the open field (left) but not MSU or MSUS females (right). (C) F1 MSUS males made fewer attempts to enter the open field (left) but not MSU or MSUS females (right). (D) F1 MSU and MSUS males and females covered similar distances in an open field. Males: MSU, $n=6$, MSUS, $n=8$, control, $n=8$; females: MSU, $n=7$, MSUS, $n=7$, control, $n=6 .{ }^{*} p<0.05$. Bar graphs represent mean values \pm SEM.

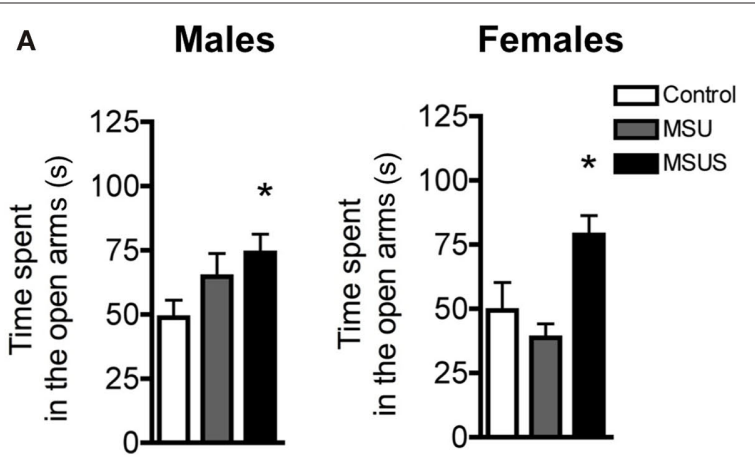

B
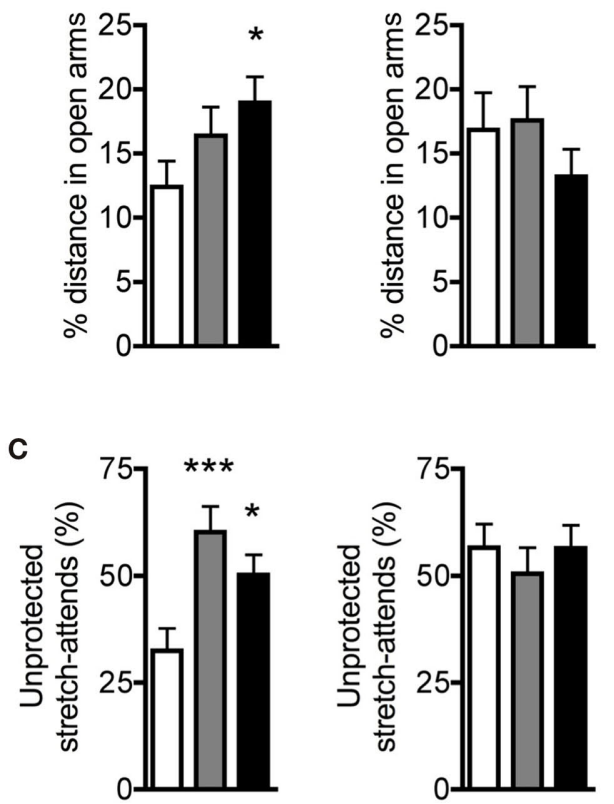

FIGURE 4 | F1 control, MSU, and MSUS mice behavior in the elevated plus maze. (A) F1 MSU and MSUS males demonstrated a graded increase in time spent in the open arms compared to control males (left). F1 MSUS females spent significantly more time in the open arms than control and MSU mice (right). (B) F1 MSUS males covered an increased proportion of their total distance in the open arm but there was no significant effect in F1 MSU or MSUS females. (C) F1 MSU and MSUS mice had an increased proportion of unprotected stretch attends (left). There was no effect in F1 MSU or MSUS females (right). Males: MSU, $n=13$, MSUS, $n=13$, control, $n=16$; females: MSU, $n=7$, MSUS, $n=9$, control, $n=7$. ${ }^{* *} p<0.001$; ${ }^{*} p<0.05$. Bar graphs represent mean values \pm SEM.

altered to the same extent. Overall, MSUS animals again showed more pronounced behavioral alterations than MSU animals, confirming that MSUS has the most severe impact on behavior.

\section{TRANSMISSION OF BEHAVIORAL ALTERATIONS TO THE OFFSPRING BY} F1 MSUS FEMALES

To examine whether these behavioral traits were heritable and could be transmitted to the following offspring, we bred adult F1 MSUS and control females with wild-type males, and generated F2 MSUS offspring under normal rearing conditions (no MS or stress). The sex ratio (male/female) and number of pups per litter were assessed and found to be similar in all groups 
(Tables 4 and 5). The ability of F1 MSUS females to raise either their own pups, or cross-fostered pups from control dams was monitored. The body weight of the F2 offspring was measured throughout development to evaluate rearing conditions. F2 control pups raised by $F 1$ control or by MSUS dams had similar weight (Figure 5A), indicating no gross difference in rearing between control and MSUS mothers. Consistently, F1 MSUS females provided adequate maternal care not only to their own biological offspring but also to the offspring of control dams after cross-fostering (Figures 5C,D). F2 MSUS pups had however reduced body weight when cross-fostered to control dams, a reduction that remained into adulthood (Figures 5A,B). This reduction was not due to any obvious alteration in maternal care provided by control dams (Figures 5C,D), suggesting the possibility that it may result from an altered response of the pups to the stress of cross-fostering.

Despite being properly raised, F2 MSUS pups had behavioral alterations comparable to their $F 1$ biological dams. The male offspring of F1 MSUS dams, whether raised by control or by MSUS dams, had reduced latency to enter unfamiliar areas in the free exploratory paradigm [pup effect: $F(1,53)=4.52, p<0.05$; Figure 6A] . F2 MSUS males also spent more time in the open arms of the elevated plus maze [pup effect: $F(1,55)=4.54, p<0.05$ ] and made more unprotected stretch-attend postures [pup effect: $F(1$, 58) $=5.38, p<0.05$; Figures 6B-D].

Similar behavioral alterations were observed in F2 MSUS females, although to a lesser extent [\% distance, pup effect: $F(1,55)=14.152, p<0.001$; unprotected stretch attends, pup effect: $F(1,59)=3.82, p=0.056$; Figures 6B-D], suggesting the possibility of a sex-dependent effect. Interestingly, the abnormal behavioral traits were not due to rearing by an MSUS dam, since F1 MSUS dams had normal maternal behaviors and control pups cross-fostered to these dams had no behavioral alterations (Figure 5). These results overall suggest that non-genomic mechanisms underlie the transmission of the behavioral abnormalities. Notably, since some of the behavioral deficits in F2 MSUS males could sometimes be corrected by rearing by a control dam [interaction: $F(1,55)=6.18$, $p<0.05$; Figure 6B], additional environmental factors besides maternal care may also have an influence. These factors have not been identified but may include social conditions, physiological or nutritional differences.

\section{IMPACT OF THE MANIPULATION ON STRESS MEDIATORS}

We next tested the impact of MSUS on functional indicators of the HPA axis, and used corticosterone in plasma as one of these indicators. We observed that the level of plasma corticosterone was normal in both basal and stress-induced conditions in F1 MSU and MSUS mice (Figure 7A). Likewise, the weight of adrenal glands was comparable in all groups, indicating no gross alteration of the HPA axis in the periphery (Figure 7B). We next examined HPA markers in the brain and focused on CRFR1 and 2, two major receptors associated with the stress response. The level of CRFR 1 and 2 in the brain of $F 1$ control and MSUS animals was measured by autoradiography. While CRFR1 binding was not changed in any of the examined areas, i.e., hippocampus, amygdala, hypothalamus, CRFR2 binding was significantly decreased in several nuclei of the amygdala and the hypothalamus, but not in cortical areas [LH: $t(17)=2.31$,
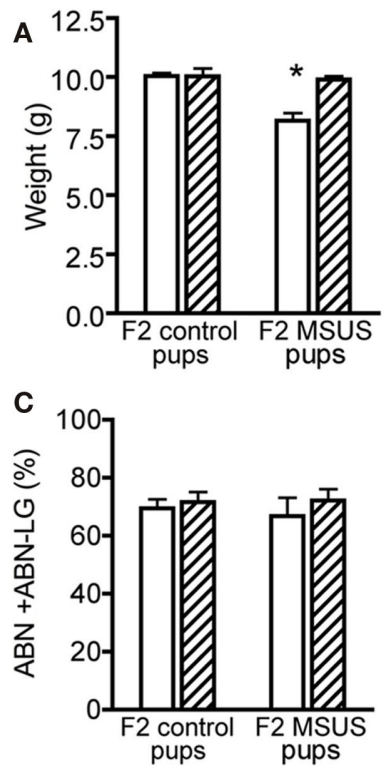

FIGURE 5 | Postnatal development in $\boldsymbol{F} 2$ offspring. (A,B) Control pups cross-fostered to MSUS dams had normal body weight at (A) weaning ( $n=28$ ) and $(\mathbf{B})$ in adulthood $(n=29$ ), but $F 2$ MSUS pups cross-fostered to $F 2$ control dams had decreased body weight at $(\mathbf{A})$ weaning $(n=27)$ and $(\mathbf{B})$ in adulthood ( $n=25)$, compared to control pups raised with their biological dams (weaning, $n=92$; adult, $n=89$ ) and MSUS pups raised with their biological dams
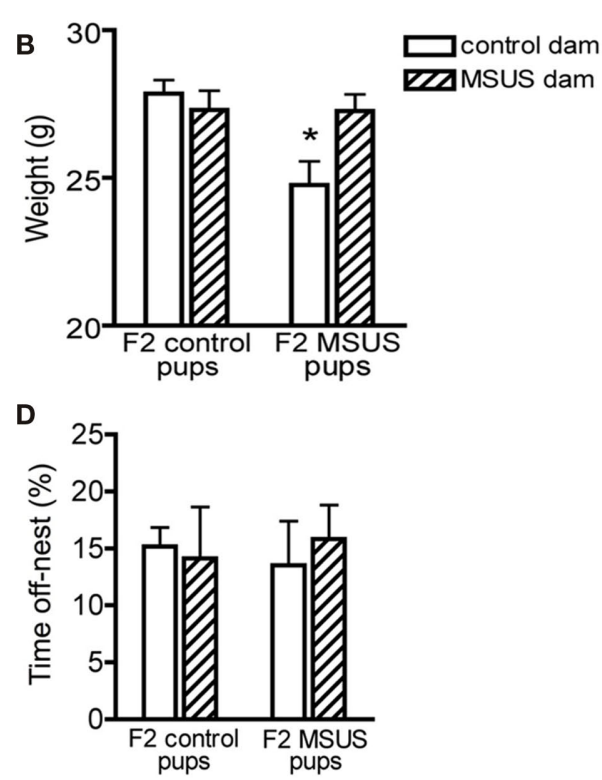

(weaning, $n=51$; adult, $n=55$ ). (C,D) Control and MSUS dams provided a similar level of (C) active maternal care [arched-back nursing and arched-back nursing/licking-grooming ( $A B N+A B N-L G)]$ and (D) time spent off-nest, whether caring for their biological offspring (control, $n=12$; MSUS, $n=9$ ) or crossfostered pups (control, $n=3$; MSUS, $n=3$ ). ${ }^{*} p<0.05$. Bar graphs represent mean values \pm SEM. 
A

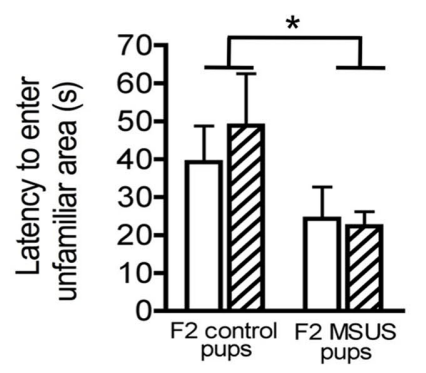

B

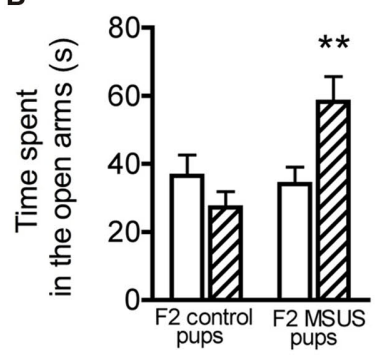

C

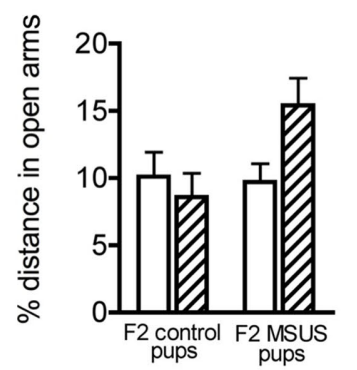

D

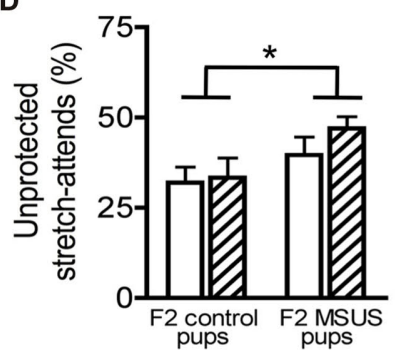

FIGURE 6 | Behavior of $F \mathbf{2}$ mice in the free exploratory paradigm and elevated plus maze. (A) F2 MSUS males entered the unfamiliar partitions of the free exploratory paradigm sooner than control mice, irrespective of the treatment of the dam that reared them. No change was observed in F2 MSUS females. (B) F2 MSUS males reared by their biological dams spent more time in the open arms than control mice, however when raised with a control dam, this behavioral deficit was reversed. No change was observed in F2 MSUS females. (C) No significant difference in distance covered in the open arms was observed in $\mathrm{F} 2$ males. However, MSUS pups raised by their biological dams had a strong tendency to cover a larger proportion of total distance in the open arms. F2

\section{Females}

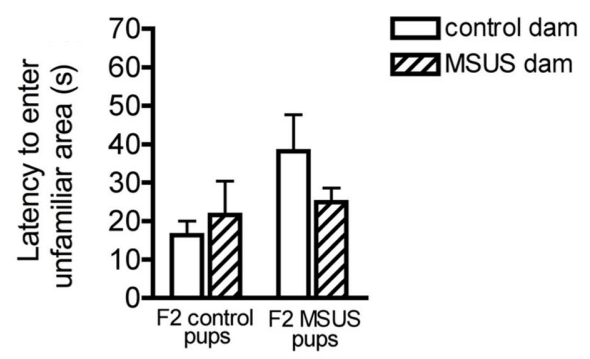

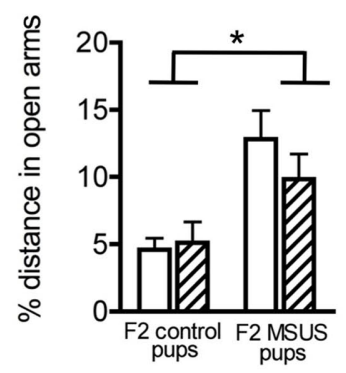

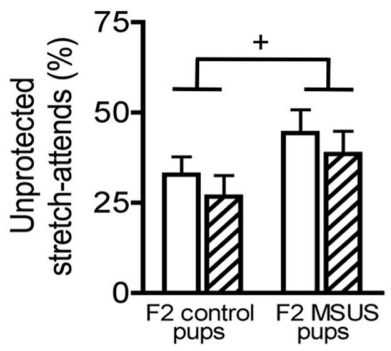

MSUS females also covered a greater proportion of total distance in the open arms, irrespective of the treatment received by their dam. (D) F2 MSUS mice had a greater proportion of stretch attends from an unprotected area than control mice, irrespective of the treatment received by the dam that reared them. A strong trend for similar behavioral alterations was observed in F2 MSUS females when compared to control. Control pups raised by biological dam, males $n=20$, females $n=16$; MSUS pups raised with biological dam, males $n=18$, females $n=14$; control pups raised by MSUS dam, males $n=14$, females $n=15$; MSUS pups raised with control dam, males $n=11$, females $n=16.0 .05<+<0.1$; ${ }^{*} p<0.05 ;{ }^{* *} p<0.01$. Bar graphs represent mean values \pm SEM.

$p<0.05$, PVN: $t(16)=2.67, p<0.05$, BMA: $t(16)=2.13, p<0.05$, $\mathrm{MePV} / \mathrm{D}: t(16)=2.67, p<0.05$; Figure 8]. These results suggest that CRFR2 may be an important mediator of the effect of early stress.

\section{DISCUSSION}

Early postnatal stress is known to induce behavioral alterations during adulthood in rodents, primates, and humans. Here, we show that in mice, unpredictable MS combined with maternal stress 

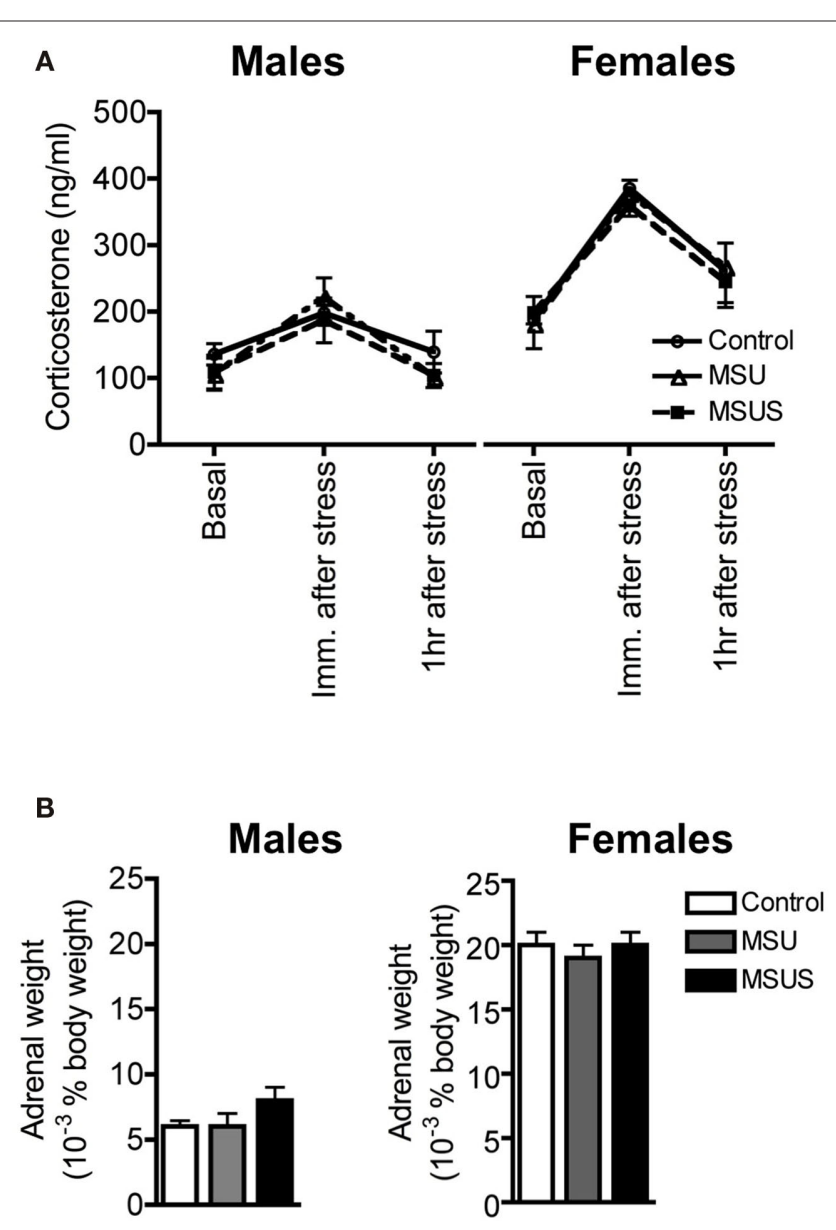

FIGURE 7 |Assessment of markers of the HPA axis in F1 control, MSU, and MSUS mice. (A) All groups had a comparable titer of corticosterone in plasma 1 week before, immediately after, and $1 \mathrm{~h}$ after a 20-min restraint stress (all measures were performed in the same animals across treatment). Males: MSU, $n=4$, MSUS, $n=4$, control, $n=6$; females, MSU, $n=4$, MSUS, $n=9$, control, $n=5$. (B) Similar adrenal weight in all mice. Males MSU, $n=6$, MSUS, $n=8$, control, $n=8$; females: MSU, $n=7$, MSUS, $n=8$, control, $n=7$. Bar graphs represent mean values \pm SEM

persistently alters behavioral responses in aversive and unfamiliar environments in the separated animals when adult, and makes the animals unable to carefully evaluate unknown territories, and assess risk. We show further, that these alterations are transmitted to the following generation even in the absence of any further exposure to stress. Our data indicate that the mild behavioral deficits resulting from unpredictable MS alone are more pronounced when separation is aggravated by concomitant unpredictable maternal stress, suggesting that stress has a cumulative effect on behavioral responses. Unpredictability of MS is also likely to exacerbate the behavioral defects, consistent with the previous observation that chronic stress in adult rats or mice is more detrimental when unpredictable than when predictable (Haile et al., 2001).

Notably in our model, MSUS alters behavior primarily in males (although females also have some behavioral deficits), consistent with previous findings that males are more susceptible to the detrimental effects of early stress than females (Holmes et al., 2005; Kikusui and Mori, 2009). The fact that dams show increased maternal care toward their male offspring may partially explain why deficits in maternal care induced by MS have a greater effect on the male brain (Alleva et al., 1989). Although a potential effect of hormonal cycles on behavior cannot be excluded in females, behavioral responses were not more variable in females (Control, MSU, or MSUS) than in males, indicating no major influence of the manipulation on female behavior. Importantly, despite their relatively mild behavioral alterations, F1 MSUS females do transmit these alterations to their offspring. Transmission is independent of maternal care or other environmental factors, suggesting the involvement of non-genomic mechanisms. The fact that some alterations can be transmitted without being expressed by the mother herself suggests that mice can act as "silent" or asymptomatic carriers of these traits, as was demonstrated in males (Franklin et al., 2010). This has also been reported in humans, but the mechanisms remain unknown (Pembrey et al., 2006; Roseboom et al., 2006; Sterba et al., 2007; Vige et al., 2008; Kim et al., 2009). Overall, these findings significantly extend recent data showing that F1 MSUS males transmit their behavioral defects across several generations (Franklin et al., 2010). They provide evidence that not only innate behaviors, but also behavioral alterations induced by unfavorable environmental conditions can be inherited.

Previous findings in our lab have suggested that the increased exploration of aversive environments by MSUS mice reflects a deficit in behavioral control rather than an overall reduction in anxiety, or increased emotional blunting (Franklin et al., 2010). These findings demonstrated that the behavioral deficits in MSUS mice are observed only when the aversive areas are unfamiliar, but are not observed when the aspect of novelty is removed. Thus, MSUS appears to impair an animal's ability to properly evaluate danger associated with unfamiliarity or novelty, and causes inadequate impulsive-prone responses in both, secure and insecure environments. However, the possibility that these behaviors are associated with some aspects of reduced anxiety cannot be ruled out. Furthermore, these behavioral alterations are similar in males and females (although more pronounced in males), but may be revealed differently depending on the task and parameter examined. Interestingly, MSUS treatment affects the dams similarly to their offspring, albeit to a lesser extent. The behavior of F0 MSUS dams on the elevated plus maze was only mildly perturbed despite the severity of the combination between MS and maternal stress treatment, suggesting that little effects, if any, would be present in F0 MSU dams. However, this has not been assessed at this time.

The molecular mechanisms underlying the behavioral alterations induced by MSUS are not known. Our results show normal CRFR1 expression but reduced CRFR2 expression in amygdala nuclei, LH, and PVN, suggesting a specific perturbation of CRFR2dependent stress pathways in MSUS mice. It is also possible that F1 MSU also have deficient CRFR2 binding but this was not examined. However, because F1 MSU mice have a relatively mild behavioral phenotype, it is unlikely that significant changes in CRFR2 binding would be observed. CRFR2 is a receptor for CRF-related stress peptides thought to promote the adaptation and recovery from stressful events, that when activated is anxiolytic (de Kloet et al., 2005). In contrast, CRFR1 is involved in the rapid "fight or flight" response to stress and involves the activation of the HPA axis (de Kloet et al., 2005). A reduction in CRFR2 in key regions 


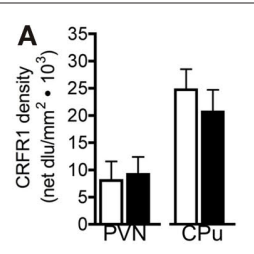

B

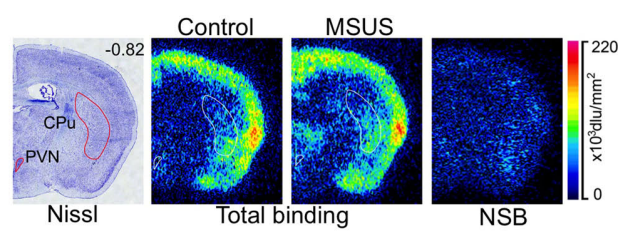

D
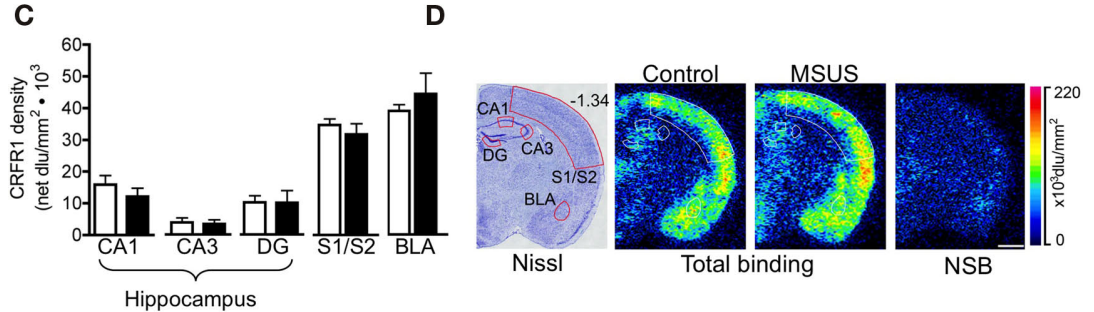

$\mathbf{F}$
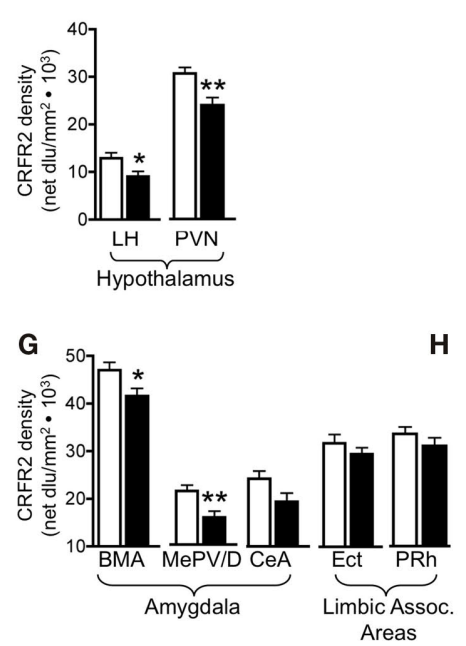

H

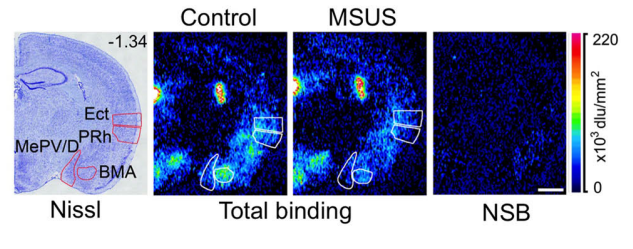

FIGURE 8 | Corticotropin releasing factor receptor binding in F1 MSUS and control mice. $(\mathbf{A}, \mathbf{C})$ Normal CRFR1 binding was observed in several brain areas in F1 MSUS brain (MSUS, $n=5-6$, control, $n=5-6)$. (E,G) Reduced CRFR2 binding was observed in several hypothalamic and amygdala subnuclei, but not in cortical areas in F1 MSUS brain (MSUS, $n=12$, control, $n=6-7$ ). (B,D,F,H) Sections showing Nissl staining (B,D) total CRFR1 binding and CRFR1 non-specific binding (NSB), and (F,H) total CRFR2 binding and CRFR2 NSB, in different brain areas in $\mathrm{F} 1$ control and MSUS mice. Number in top right corner of Nissl section indicates Bregma position. Scale bar $1 \mathrm{~mm} .{ }^{*} p<0.05 ;{ }^{*} p<0.01$. $\mathrm{BLA}$, basolateral amygdala; BMA, basomedial amygdala; $\mathrm{CPu}$, caudate putamen; DG, dentate gyrus; Ect, ectorhinal cortex; LH, lateral hypothalamus; PVN, paraventricular nucleus; MePV/D, medial posteroventral and medial posterodorsal amygdala; PRh, perirhinal cortex; S1/S2, somatosensory cortex. Bar graphs represent mean values \pm SEM. involved in the stress response is therefore likely to contribute to the altered behavioral response to aversive environments induced by MSUS. In line with this suggestion, pharmacological antagonists of CRFR2 have been shown to increase exploratory activity in aversive environments in mice (Pelleymounter et al., 2002, 2004). Other measures of the stress pathways including the level of resting and stress-induced plasma corticosterone, and the weight of adrenal glands were also normal, in accordance with previous reports showing that only extended periods of MS (over $4 \mathrm{~h}$ ) notably affect the HPA axis in mice (Schmidt et al., 2004). They also corroborate findings in humans showing a dissociation between HPA response and prolonged stress or trauma, indicating that the HPA axis is not necessarily over-activated after early stress (Gunnar and Vazquez, 2001). However, while there is a dissociation between the susceptibility of two key stress pathways to MSUS, we suggest that CRFR2 signaling is only one of several systems that may be affected. MSUS is a severe and general manipulation that likely affects multiple pathways, resulting in the complex and sex-specific behavioral phenotype as observed. A pharmacological manipulation of any single component of these pathways is therefore unlikely to reverse the observed behavioral phenotype.

Previous research has shown that the quality of maternal care determines an individual's behavior later in life (Weaver, 2009; Curley et al., 2010). In our model, while maternal care was altered by MSUS in F0 females and led to behavioral alterations in F1 offspring, it was not perturbed in $F 1$ females and is therefore not responsible for the transmission of the behavioral alterations to F2 offspring. This is supported by our recent demonstration that the behavioral alterations can also be transmitted by F1 MSUS males, and further by F2 males to F3 offspring (Franklin et al., 
2010). Together, these findings strongly suggest that epigenetic mechanisms are likely to underlie the transgenerational inheritance of behavioral abnormalities induced by early stress, and that these mechanisms may be at work similarly in males and females. Interestingly, while transmission through MSUS males tended to affect the female offspring (Franklin et al., 2010), transmission through MSUS females presented here preferentially affects male offspring. This parallels a recent study showing an association between paternal open field activity and open field activity of female, but not male offspring (Alter et al., 2009). The mechanisms for this bias toward transmission to offspring of the other sex are unknown. However, parent-of-origin susceptibility to the transmission of epigenetic profiles was previously suggested by sex-dependent effects of imprinted genes (Hager et al., 2008; Curley et al., 2010). While such sex-linked epigenetic mechanisms have not yet been determined, sex-dependent trans control of expression, like for instance, differential expression at a sex-specific modifier locus during gametogenesis, has been suggested (Hager et al., 2008).

Cross-fostering experiments in the $F 2$ generation also showed that some of the behavioral abnormalities could be erased in F2 MSUS pups when raised by $F 1$ control dams. This was not due to altered maternal care since F1 MSUS dams provided a level of care comparable to control dams and could properly raise control pups. However, subtle changes in physiological or social parameters such as milk content or quality, or altered social interactions cannot be excluded. Alternatively, the act of cross-fostering itself may modify or erase some of the epigenetic marks underlying the behavioral defects. The fact that F2 MSUS pups raised by $F 1$ control dams, but not $F 2$ control pups raised by F1 MSUS dams, had an immediate decrease in weight that persisted into adulthood indicates that F2 MSUS mice did react to cross-fostering itself, suggesting the possibility that some epigenetic marks may have been disrupted as well. Finally, these results suggest that the behavioral alterations induced by MSUS are still labile at an early stage of development, and can be corrected.

In humans, the inability to properly respond to danger and evaluate one's environment, and impaired behavioral control, are major symptoms of several behavioral and psychiatric disorders such as personality disorders, antisocial behaviors, attention deficit/

\section{REFERENCES}

Alleva, E., Caprioli, A., and Laviola, G. (1989). Litter gender composition affects maternal behavior of the primiparous mouse dam (Mus musculus). J. Comp. Psychol. 103, 83-87.

Alter, M. D., Gilani, A. I., Champagne, F. A., Curley, J. P., Turner, J. B., and Hen, R. (2009). Paternal transmission of complex phenotypes in inbred mice. Biol. Psychiatry 66, 1061-1066.

Birke, L. I., and Sadler, D. (1986). Patterns of exploratory behavior in the spiny mouse, Acomys cahirinus. Behav. Neural Biol. 45, 88-106.

Caldji, C., Diorio, J., and Meaney, M. J. (2000). Variations in maternal care in infancy regulate the development of stress reactivity. Biol. Psychiatry 48, 1164-1174.

hyperactivity disorder (ADHD), and drug addiction (Hinshaw, 2003). These defects are manifested differently depending on an individual's genetic make-up, physiological and emotional state, and life history. The mouse model presented here does not fully recapitulate these disorders in their entirety, but does express specific behavioral traits associated with these disorders, It may therefore be useful to better understand the neural and epigenetic mechanisms involved in the etiology of such human diseases, and open new perspectives for potential novel therapeutic approaches. The possibility that non-genomic mechanisms may underlie the transmission of complex behaviors across generations, perhaps as a result of inheritance of epigenetic marks induced by environmental stimuli, has important implications for the incidence rate of diseases, and may help explain the complex and often highly prevalent profile of inheritance of many complex diseases in humans.

In conclusion, this paper demonstrates that the combination of unpredictable MS with maternal stress more severely affects behavior than unpredictable MS alone. The manipulation induces behavioral defects that are transmitted to the following generation, independent of maternal care. In F1 mice, it correlates with a dissociation between CRFR1- and CRFR2-dependent signaling pathways, and affects CRFR2 only. The MSUS paradigm is therefore a useful novel model to investigate the long-term and persistent effects of severe stress in rodents, and as a model to elucidate the mechanisms underlying non-genomic transmission of behavioral alterations across generations.

\section{ACKNOWLEDGMENTS}

Harma Feitsma, Makeba Kampara, Steffi Lehmann, Annegret Lesslauer, Laurie Paroz, Sandrine Romand, Dietmar Benke, Florence Crestani, Adrian Fairburn, Astrid Sasse, Hans-Rüdi Olpe, Andreas Lüthi, and Martin Bohus for discussions in the first phase of the project, Gregor Fisher for help with the mouse colony. The lab is supported by the University of Zürich, the Swiss Federal Institute of Technology, the Swiss National Science Foundation, the National Center for Competence in Research "Neural Plasticity and Repair," the Human Frontier Science Program, the Borderline Personality Disorder Research Foundation, Roche, Novartis Research Foundation.

behaviour and on the total neuron number in the mouse hippocampus. Brain Struct. Funct. 212, 403-416.

Franklin, T. B., Russig, H., Weiss, I. C., Graff, J., Linder, N., Michalon, A., Vizi, S., and Mansuy, I. M. (2010). Epigenetic transmission of the impact of early stress across generations. Biol. Psychiatry 68, 408-415.

Griebel, G., Belzung, C., Misslin, R., and Vogel, E. (1993). The free exploratory paradigm: an effective method for measuring neophobic behaviour in mice and testing potential neophobia reducing drugs. Behav. Pharmacol. 4, 637-644.

Gunnar, M. R., and Vazquez, D. M. (2001). Low cortisol and a flattening of expected daytime rhythm: potential indices of risk in human development. Dev. Psychopathol. 13, 515-538.
Hager, R., Cheverud, J. M., Leamy, L. J., and Wolf, J. B. (2008). Sex dependent imprinting effects on complex traits in mice. BMC Evol. Biol. 8, 303. doi: 10.1186/1471-2148-8-303

Haile, C. N., GrandPre, T., and Kosten, T. A. (2001). Chronic unpredictable stress, but not chronic predictable stress, enhances the sensitivity to the behavioral effects of cocaine in rats. Psychopharmacology (Berl.) 154, 213-220.

Heim, C., Mletzko, T., Purselle, D., Musselman, D. L., and Nemeroff, C. B. (2008). The dexamethasone/corticotropin-releasing factor test in men with major depression: role of childhood trauma. Biol. Psychiatry 63, 398-405.

Hinshaw, S. P. (2003). Developmental psychopathology: specificity versus 
generality of linkages. Ann. N. Y. Acad. Sci. 1008, 149-159.

Hirshfeld-Becker, D. R., Biederman, J., Faraone, S. V., Segool, N., Buchwald, J., and Rosenbaum, J. F. (2004). Lack of association between behavioral inhibition and psychosocial adversity factors in children at risk for anxiety disorders. Am. J. Psychiatry 161, 547-555.

Holmes, A., le Guisquet, A. M., Vogel, E., Millstein, R.A., Leman, S., and Belzung, C. (2005). Early life genetic, epigenetic and environmental factors shaping emotionality in rodents. Neurosci. Biobehav. Rev. 29, 1335-1346.

Huot, R. L., Gonzalez, M. E., Ladd, C. O., Thrivikraman, K. V., and Plotsky, P. M. (2004). Foster litters prevent hypothalamic-pituitary-adrenal axis sensitization mediated by neonatal maternal separation. Psychoneuroendocrinology 29, 279-289.

Huot, R. L., Thrivikraman, K. V., Meaney, M. J., and Plotsky, P. M. (2001). Development of adult ethanol preference and anxiety as a consequence of neonatal maternal separation in long Evans rats and reversal with antidepressant treatment. Psychopharmacology (Berl.) 158, 366-373.

Iversen, A. C., Fear, N. T., Simonoff, E., Hull, L., Horn, O., Greenberg, N., Hotopf, M., Rona, R., and Wessely, S. (2007). Influence of childhood adversity on health among male UK military personnel. Br. J. Psychiatry 191, 506-511.

Jaffee, S. R., Moffitt, T. E., Caspi, A., Fombonne, E., Poulton, R., and Martin, J. (2002). Differences in early childhood risk factors for juvenileonset and adult-onset depression. Arch. Gen. Psychiatry 59, 215-222.

Kikusui, T., and Mori, Y. (2009). Behavioural and neurochemical consequences of early weaning in rodents. J. Neuroendocrinol. 21, 427-431.

Kim, H. K., Capaldi, D. M., Pears, K. C., Kerr, D. C., and Owen, L. D. (2009). Intergenerational transmission of internalising and externalising behaviours across three generations: genderspecific pathways. Crim. Behav. Ment. Health 19, 125-141.

Lehmann, J., Russig, H., Feldon, J., and Pryce, C. R. (2002). Effect of a single maternal separation at different pup ages on the corticosterone stress response in adult and aged rats. Pharmacol. Biochem. Behav. 73 , 141-145.

Lister, R. G. (1987). The use of a plusmaze to measure anxiety in the mouse. Psychopharmacology 92, 180-185.

Liu, D., Diorio, J., Day, J. C., Francis, D. D., and Meaney, M. J. (2000). Maternal care, hippocampal synaptogenesis and cognitive development in rats. Nat. Neurosci. 3, 799-806.

Mathieu, G., Denis, S., Lavialle, M., and Vancassel, S. (2008). Synergistic effects of stress and omega-3 fatty acid deprivation on emotional response and brain lipid composition in adult rats. Prostaglandins Leukot. Essent. Fatty Acids 78, 391-401.

McIntosh, J., Anisman, H., and Merali, Z. (1999). Short- and long-periods of neonatal maternal separation differentially affect anxiety and feeding in adult rats: gender-dependent effects. Brain Res. Dev. Brain Res. 113, 97-106.

Moffitt, T. E., Caspi, A., Harrington, H., Milne, B. J., Melchior, M., Goldberg, D., and Poulton, R. (2007). Generalized anxiety disorder and depression: childhood risk factors in a birth cohort followed to age 32. Psychol. Med. 37, 441-452.

Murgatroyd, C., Patchev, A. V., Wu, Y., Micale, V., Bockmuhl, Y., Fischer, D., Holsboer, F., Wotjak, C. T., Almeida, O. F., and Spengler, D. (2009). Dynamic DNA methylation programs persistent adverse effects of early-life stress. Nat. Neurosci. 12, 1559-1566.

Neigh, G.N., Gillespie, C.F., and Nemeroff, C. B. (2009). The neurobiological toll of child abuse and neglect. Trauma Violence Abuse 10, 389-410.

Parfitt, D. B., Levin, J. K., Saltstein, K. P., Klayman, A. S., Greer, L. M., and Helmreich, D. L. (2004). Differential early rearing environments can accentuate or attenuate the responses to stress in male C57BL/6 mice. Brain Res. 1016, 111-118.

Paxinos, G., and Franklin, K. B. J. (2001). The Mouse Brain in Stereotaxic Coordinates, 2nd Edn. San Diego: Academic Press.

Pelleymounter, M. A., Joppa, M., Ling, N., and Foster, A. C. (2002). Pharmacological evidencesupporting a role for central corticotropin-releasing factor(2) receptors in behavioral, but not endocrine, response to environmental stress. J. Pharmacol. Exp. Ther 302, 145-152.

Pelleymounter, M. A., Joppa, M., Ling, N., and Foster, A. C. (2004). Behavioral and neuroendocrine effects of the selective CRF2 receptor agonists urocortin II and urocortin III. Peptides 25, 659-666.

Pellow, S., Chopin, P., File, S. E., and Briley, M. (1985). Validation of open: closed arm entries in an elevated plus-maze as a measure of anxiety in the rat. $J$. Neurosci. Methods 14, 149-167.

Pembrey, M. E., Bygren, L. O., Kaati, G., Edvinsson, S., Northstone, K., Sjostrom, M., and Golding, J. (2006). Sex-specific, male-line transgenerational responses in humans. Eur. J. Hum. Genet. 14, 159-166.

Quartermain, D., Stone, E. A., and Charbonneau, G. (1996). Acute stress disrupts risk assessment behavior in mice. Physiol. Behav. 59, 937-940.

Rikhye, K., Tyrka, A. R., Kelly, M. M., Gagne, G. G. Jr., Mello, A. F., Mello, M. F., Price, L. H., and Carpenter, L. L. (2008). Interplay between childhood maltreatment, parental bonding, and gender effects: impact on quality of life. Child Abuse Negl. 32, 19-34.

Rodgers, R. J., and Dalvi, A. (1997). Anxiety, defence and the elevated plus-maze. Neurosci. Biobehav. Rev. 21, 801-810.

Roman, E., Gustafsson, L., Berg, M., and Nylander, I. (2006). Behavioral profiles and stress-induced corticosteroid secretion in male Wistar rats subjected to short and prolonged periods of maternal separation. Horm. Behav. 50, 736-747.

Roseboom, T., de Rooij, S., and Painter, R. (2006). The Dutch famine and its long-term consequences for adult health. Early Hum. Dev. 82, 485-491.

Schmidt, M., Enthoven, L., van Woezik, J. H., Levine, S., de Kloet, E. R., and Oitzl, M. S. (2004). The dynamics of the hypothalamic-pituitary-adrenal axis during maternal deprivation. $J$. Neuroendocrinol. 16, 52-57.

Shamir-Essakow, G., Ungerer, J. A., and Rapee, R. M. (2005). Attachment, behavioral inhibition, and anxiety in preschool children. J. Abnorm. Child. Psychol. 33, 131-143.
Slotten, H. A., Kalinichev, M., Hagan, J. J., Marsden, C. A., and Fone, K.C. (2006) Long-lasting changes in behavioural and neuroendocrine indices in the rat following neonatal maternal separation: gender-dependent effects. Brain Res. 1097, 123-132.

Sterba, S. K., Prinstein, M. J., and Cox, M. J. (2007). Trajectories of internalizing problems across childhood: heterogeneity, external validity, and gender differences. Dev. Psychopathol. 19, 345-366.

Teixeira-Silva, F., Dias Antunes, F., Santos Silva, P. R., Goes, T. C., Dantas, E. C., Santiago, M. F., and Machado de Andrade, R. (2009). The free-exploratory paradigm as a model of trait anxiety in rats: test-retest reliability. Physiol. Behav. 96, 729-734.

Tezval, H., Jahn, O., Todorovic, C., Sasse, A., Eckart, K., and Spiess, J. (2004). Cortagine, a specific agonist of corticotropin-releasing factor receptor subtype 1 , is anxiogenic and antidepressive in the mouse model. Proc. Natl. Acad. Sci. U.S.A. 101, 9468-9473.

Vige, A., Gallou-Kabani, C., and Junien, C. (2008). Sexual dimorphism in non-Mendelian inheritance. Pediatr. Res. 63, 340-347.

Weaver, I. C. (2009). Shaping adult phenotypes through early life environments. Birth Defects Res. C Embryo Today 87, 314-326.

Conflict of Interest Statement: The authors declare that the research was conducted in the absence of any commercial or financial relationships that could be construed as a potential conflict of interest.

Received: 20 October 2010; accepted: 17 January 2011; published online: 04 February 2011.

Citation: Weiss IC, Franklin TB, Vizi $S$ and Mansuy IM (2011) Inheritable effect of unpredictable maternal separation on behavioral responses in mice. Front. Behav. Neurosci. 5:3. doi: 10.3389/ fnbeh.2011.00003

Copyright $\odot 2011$ Weiss, Franklin, Vizi and Mansuy. This is an open-access article subject to an exclusive license agreement between the authors and Frontiers Media SA, which permits unrestricted use, distribution, and reproduction in any medium, provided the original authors and source are credited. 